\title{
DESPECKLING ALGORITHMS FOR REMOVING NOISE IN MEDICAL IMAGES
}

\author{
D.Devasena $^{1}$, K.Srinivasan ${ }^{2}$, B.Sharmila ${ }^{3}$, A. Booja ${ }^{4}$ \\ ${ }^{1}$ Assistant Professor, Dept of EIE, Sri Ramakrishna Engineering College, Coimbatore \\ ${ }^{2,3}$ Professor, Dept of EIE, Sri Ramakrishna Engineering College, Coimbatore \\ ${ }^{3}$ Student, M.E. Control and Instrumentation Engineering
}

\begin{abstract}
Article History:Received:11 november 2020; Accepted: 27 December 2020; Published online: 05 April 2021

Abstract : The medical and satellite images are mostly corrupted by a multiplicative granular noise called
speckle noise which degrades the quality of the images captured by using medical imaging techniques and also
Synthetic Aperture Radar images. It causes difficulties in image interpretation and this is mainly due to back
scattered signals from the multiple targets. In medical field, the diagnosis of the tissues, bones and organs takes
place by using imaging techniques. By using different imaging techniques, the medical images are captured and
used for diagnosis. Different types of filtering techniques are proposed in the literature to remove the speckle
noise in medical and satellite images. In this research paper different types of adaptive filters and its
modifications are proposed and compared. The filters like modified lee filter, modified Edge Enhanced lee filter,
modified fast bilateral filter and Modified Particle Swarm Optimization based despeckling algorithm. The
results are verified for both simulated images and real medical images and also for Synthetic Aperture Radar
images. The results are compared in terms of both objective and subjective analysis for simulated and real
medical images. The simulation is done using MATLAB R2013 and the visual qualities of the images are
analyzed for varying noise densities.
\end{abstract}

Keywords:

\section{INTRODUCTION}

Speckle noise is a type of multiplicative noise which corrupts SAR images, ultrasound images etc. The noises in medical images occur due to the reflected waves from the targeted organs which are captured by using different imaging devices. The different imaging devices used for capturing medical devices is Ultrasound scanning, Magnetic resonance imaging, $\mathrm{X}$ - ray imaging and Computer tomography imaging.

The filters used for the removal of speckle noise are classified as non adaptive filters and adaptive filters. The speckle denoising algorithm follows Homomorphic filtering, which converts the multiplicative noise into additive noise and then the noise reduction is done. For the removal of additional noises there are different methods like least mean squares, averaging filter, wiener filtering, wavelet based denoising methods are adopted (Stian Solbo \& Torbjorn Eltoft 2008).

Speckle reduction filters are differentiated as Scalar filters and Adaptive filters. Scalar filters are based on local statistics which removes the speckle in homogenous layer and preserve the details. The mean filter and Median filter are classified as scalar filters. The Adaptive filters, several adaption techniques are adopted to improve the performance of the filters. The common adaptive filters are proposed by Lee, Forst and Kuan. The well known despeckling algorithms were proposed by (Jong-Sen Lee 1980). The disadvantage of Lee filter is, it cannot effectively remove the speckles. An Enhanced Lee filter was proposed in (Loupas et al.1989), where in homogenous region the pixel value is replaced by the average value of the filter window and in heterogeneous layer the weighted average value is used to replace the pixel value. Darwin T Kuan et al. developed a linear minimum error filter which is more advantageous than Lee filter. The Edge Enhanced Lee filter was proposed in ( $\mathrm{Ju} \&$ Moloney 1998) which is based on ratio based edge detection used along with modified Lee filter which removes the speckles effectively at both low and high variance. In this paper adaptive filter like Adaptive Modified Lee Filter (AMLF), Adaptive Modified Kuan Filter (AMKF), Adaptive Modified Bilateral Filter (AMBF), Adaptive Modified Edge Enhanced modified Lee Filter (AMEELF) and optimised Particle Swarm Optimization Curvelet based Edge Enhanced Lee Filter (PSO EEMLF). The results are obtained for simulated and real medical images.

\section{RELATED WORKS}

An adaptive weighted median filter based on the weighted median coefficient is presented in [7]. The weighted median filter combines the property of the median filter and its smoothing characteristics but it 
introduces severe blurring in the image. Finally it leads to loss of diagnostic information. The filter proposed by Jong-Sen Lee (1980), is based on variance over an area and smoothing will be performed only if the variance is low and constant in that area. In SAR images, there are heterogeneous areas, in which the Lee filter can't process the situation.

Zhang et al. (2009) proposed despeckling using Bilateral Filter (BF). The BF is applied for removing the speckles in SAR images. The performance in terms of ENL and Edge Save Index (ESIN) were evaluated. Hiremath et al. (2011) proposed Contourlet transform for the removal of speckles in medical images. A new algorithm based on Contourlet transform which outperforms the wavelet transform based image denoising was developed in this research work. The suggested wavelets are not suited to represent the images in smooth contours. This was overcome by contourlet transform. Shivakumara Swamy \& Vani (2015) proposed a novel denoising method for removing speckles in SAR images using curvelet. The improved thresholding technique using curvelet transform was used as a despeckling algorithm. The PSO based noise removing technique was developed by Somnath Mukhopadhyay \& Mandal, (2014) proposed a denoising algorithm for detecting and filtering impulse noises in digital images based on PSO technique and removal of noises in medical images through thresholding and random search called GA.

\section{PROPOSED METHODS}

In the literature there are different types of despeckling algorithms are proposed, from the survey the adaptive filters proposed by Lee, Forst and Kuan cannot remove the speckle noise more efficiently. It removes the noise in homogenous region when compared to the heterogeneous region in images. Some modifications are done in these filters which improve the performance of the existing adaptive filters. The algorithm for the proposed modified Lee, Forst and Kuan filters are discussed in this chapter.

\subsection{Adaptive Modified Lee filter}

Lee [5] proposed modified lee filter with ratio based edge detector. In this paper an adaptive modified lee filter using Ratio of Averages (ROA) concept is proposed. In this ROA is used to identify the edges in the images. It is obtained by calculating the ratio of the neighbourhood pixels. The averages are calculated for sub window in the image, and by using these averages edge strengths are obtained. During filtering process, the region grows from the centre pixel to the entire image until the end point is reached. Local mean and local variance are determined by using the obtained neighborhood values in all directions. An iterative filter is proposed which is used for smoothening and sharpening. Finally an updated filtered value is replaced.

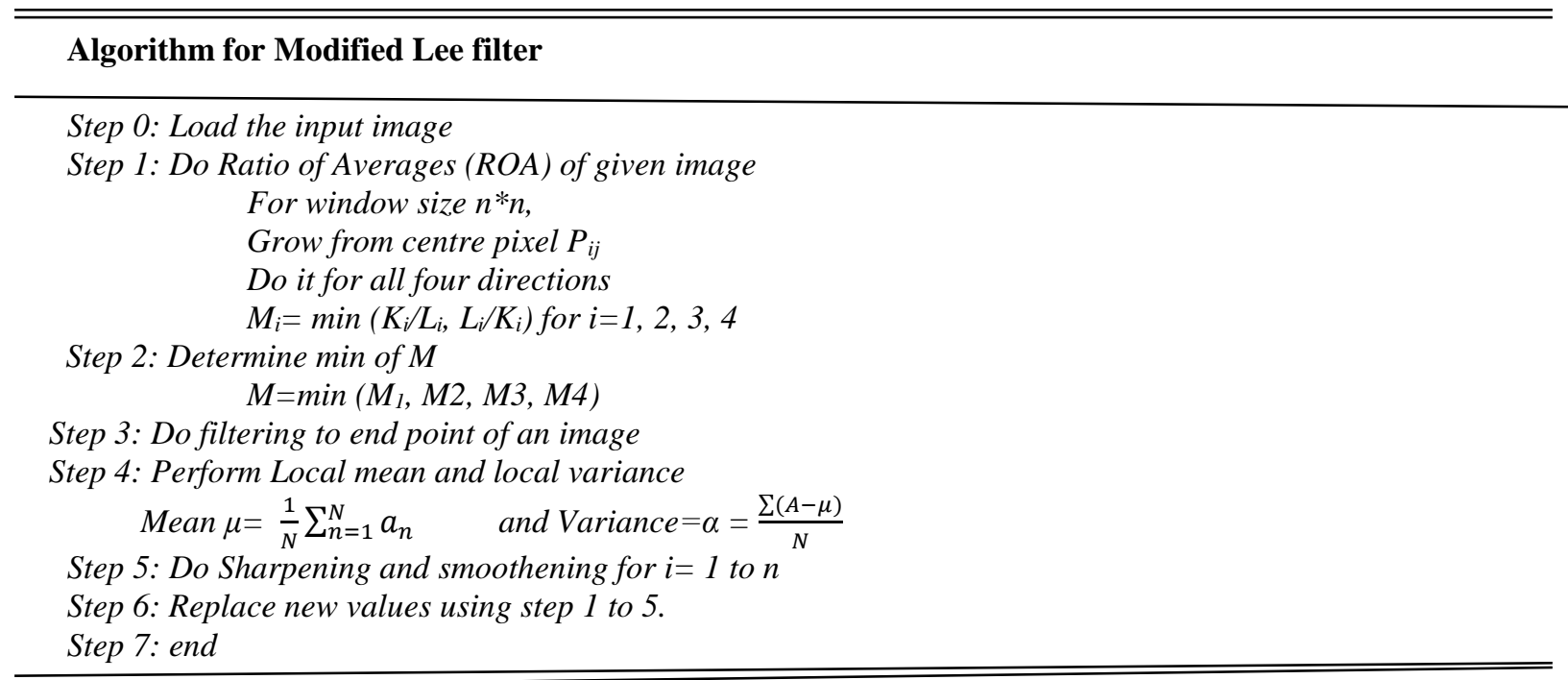

\subsection{Adaptive Modified Kuan filter}

The Kuan filter [1] uses sigmoid function to remove multiplicative noise by converting into additive noise. The sigmoid function uses weighting factor to remove noise in both homogenous and heterogeneous regions. Based on the updated weight value, the noise pixels are replaced by a new filtered pixel value. Here Minimum Mean Square Error (MMSE) and sigmoid function is used to update the weighting criteria. By applying more number of iterations, the MMSE value is reduced which in turn reduces the noise present in images.

\section{Algorithm for Adaptive Modified Kuan filter}


Step 0: Load the input image

Step 1: Calculate the noise and noise variance in the image

Noise variance $=$ std noise $*$ std noise

Step 2: Initialize the image with zeros and apply $n$ iterations

for $i=1$ :niterations

$\%$ fprintf ( rIteration \%d',i)

Step 3: Determine the weight coefficient

The weighting coefficient $c s=$ local variance/max $\left(0.0001\right.$,local mean $\left.{ }^{\wedge} 2\right)$

Step 4: calculate new image from noisy image

$f=$ localMean $+(g$-localMean $) . * k$;

for $K=\max (0,1($ noisevar. $\max (0.0000000001, \mathrm{cs})))$

Step 5: Replace new values using step 1 to 4.

Step 6: end

\subsection{Adaptive Modified Bilateral filter}

The Bilateral filter replaces every pixel by using the weighted sum of the neighboring pixels. The weighted sum of the pixels is obtained by using the similarity value and spatial distance. It also used to remove speckle noise at low frequency components. The bilateral filter is also used to preserve the edges efficiently. Initially the dynamic range of the image is identified, after the convolution kernel is used to find kernel degree. Then the Gaussian variance is obtained for the image multiplied with the convolution kernel. After this the space and range value is identified to remove the noises present in the edges. The normalized weight is calculated for the processing window and it is multiplied with the noisy pixel to get the original pixel value.

Algorithm for Adaptive Bilateral filter

Step 0: Load the input image

Step 1: Pre compute distance weight $G=\exp \left(X^{\wedge} 2+Y^{\wedge} 2\right) / 2 *$ sigma $\left.d^{\wedge} 2\right)$

Step 2: Compute Intensity weights $\left.H=\exp \left(I-A(i, j)^{\wedge} 2\right) / 2 * \operatorname{sigma} r^{\wedge} 2\right)$

Step 3: Extract local region $I=A\left(i_{\min }: i_{\max }, j_{\min }: j_{\max }\right)$

Step 4: The weight and intensity difference is calculated.

Step 5: The intensity of the pixel values $(i, j)$ and $(k, l)$ are $I(i, j)$ and $I(k, l)$ and by using smoothing parameters $\mu d$ and $\mu \mathrm{r}$.

Step 6: The normalised weight is given as $w(i, j, k, l)$ and the denoised output is given as summation of product of intensity of the pixel value and the weight of the neighboring pixel and denoised pixel to the weight of the neighboring pixel and denoised pixel.

Step 7: end

\subsection{Adaptive Edge Enhanced Modified lee filter}

The Edge Enhanced Modified Lee Filter (EEMLF) is developed which consists of two phases. First phase is used to identify the edges by using Ratio based Edge Detector. It uses Minimum Strength Pruned- Ratio of Averages (MSP-RoA) which classifies the pixel into valid region and invalid region. The threshold value is set for every direction and once the pixel reaches the value the process stops growing. The pixels inside the region are called as valid region and all other are called as Invalid region. Valid pixels then undergo statistical calculations like mean and variance. After finding edges an iterative filter is used for smoothening and sharpening. Every iteration MSP-RoA generates new edge map. The new edge map detects and identifies the edges accurately, and a denoised image is obtained fastly.

Algorithm for Edge Enhanced Modified lee filter

Step 0: Load the input image

Step 1: Do Ratio of Averages (ROA) of given image

For window size $n * n$,

Grow from centre pixel $P_{i j}$

Do it for all four directions

$M_{i}=\min \left(K_{i} / L_{i}, L_{i} / K_{i}\right)$ for $i=1,2,3,4$ 
Step 2: Calculate ENLs

Step 3: Do filtering to end point of an image

Step 4: Perform Local mean and local variance

Step 5: Do Sharpening and smoothening for $i=1$ to $n$

Step 6: Replace new values using step 1 to 5.

Step 7: end

\subsection{Particle Swarm Optimization using Curvelet Transform with Edge Enhanced Modified Lee filter (PSO-EEMLF)}

A variety of image processing algorithms and pattern classification algorithms uses optimization to find the best solution in some criteria. Optimization is one, which is used to decide a choice from a set of possibilities in order to reach a required solution with minimum error. The PSO is used to optimize the denoising coefficients [2] to filter out the noises present in images. In the speckle noise corrupted images the noisy coefficients of the denoised images are optimized by using PSO technique, and then it is filtered using Edge Enhanced Modified Lee Filter.

\section{Algorithm for PSO-EEMLF}

Step 1: Load the input noisy image (Speckle noise)

Step 2: Apply curvelet transform for the image for $J=4$ with no of scales $L=\left[\begin{array}{lll}3 & 4 & 4\end{array}\right]$

Step3: Initialize $n$ particles and fitness function, and update position and velocity of the particles.

Initialize Pbest and Gbest=0;

For $i=1$ to $n$ do

If Pbesti> pbetsnew then

Pbest=Pbesti end

Step 4: Apply inverse curvelet transform, to get an inverse image

Step5: Apply EEMLF to remove out the additional noises present in the image

Step 6: Do steps 2 to 5 to get denoised image.

\section{SIMULATION RESULTS}

The algorithm is tested over the standard test images like satellite images and medical images.. The images taken are (i) Case 189U1/Cyst (Cyst 1) (ii) Case 190U1/ Cyst ( Cyst 2) (iii) Case 192U1/Cyst (Cyst 3). The three sample MRI brain images has been taken for analysis, (i) Case bt 17 (bt 1) (ii) case bt 34 (bt 2) (iii) case D.ser6.img20 (bt 3) and two sample satellite images(SAR 1 and SAR 2) [2] \& [3]. The performance of the filter is analyzed between optimized and non optimized filters like AMLF, AMKF, AMBF, AMEELF and PSO EEMLF. The simulation results are shown for US Image and MRI image.

The real time medical images are obtained from the following websites. About 20 samples of brain MRI images (https://www.mathworks.com/matlabcentral/fileexchange/, 2 samples of ultrasound images from (https://www.ultrasound-images.com/fetal-chest/), 34 weeks baby image and ultrasound fetus image from (https://www.ultrasound-images.com/fetus-general/) are considered. The Fig. 1- 3 shows simulation results of sample simulated and real medical images, in which optimization based EEMLF shows improvement in visual quality when compared to non optimized adaptive despeckling filters.

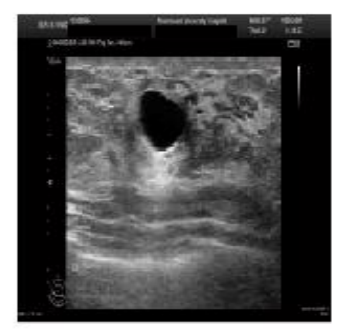

(a) Input image

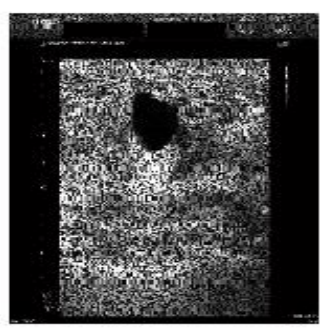

(b) Corrupted image 

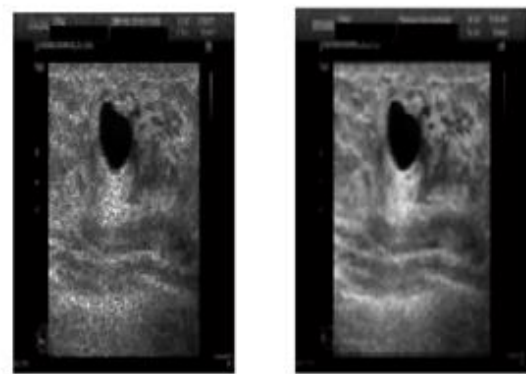

(c) AMLF

(d) AMKF

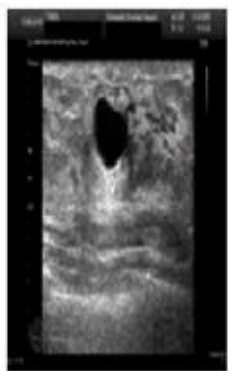

(e) AMBF

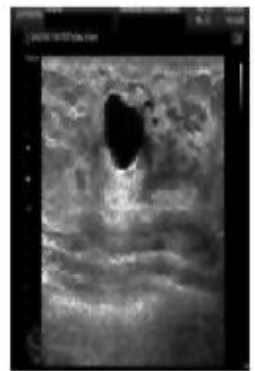

(f) AMEELF

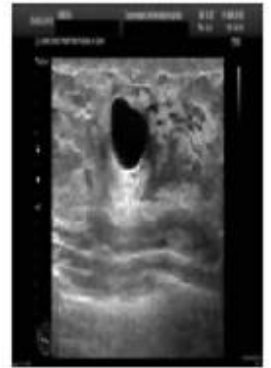

(g) PSO EEMLF

Fig 1. Simulation results of different despeckling algorithm on Cyst 1 at noise density $40 \%$ (a) Input Image (b) Corrupted image (c) AMLF (d) AMKF (e) AMBF (f) AMEELF (g) PSO EEMLF

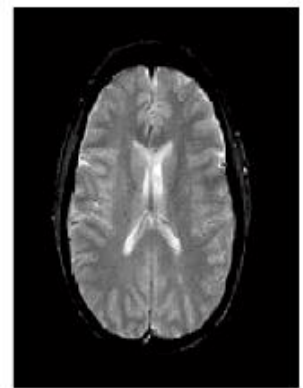

(a) Input image

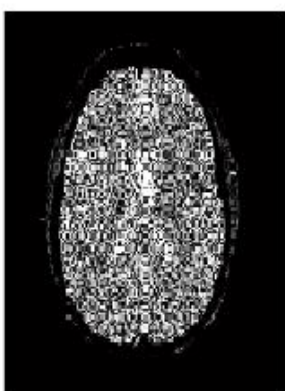

(b) Corrupted image

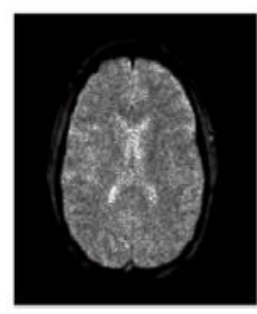

(c) AMLF

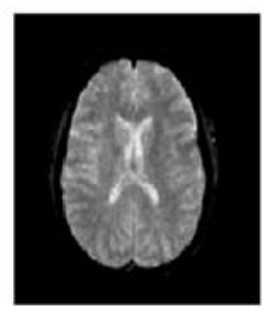

(d) AMKF

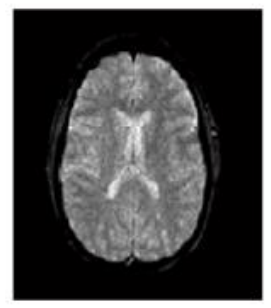

(e) AMBF

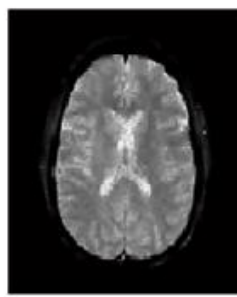

(f) AMEELF

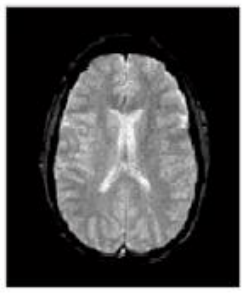

(g) PSO EEMLF

Fig 2. Simulation results of different despeckling algorithm on bt 3 at noise density 40\%((a) Input Image (b) Corrupted image (c) AMLF (d) AMKF (e) AMBF (f) AMEELF (g) PSO EEMLF

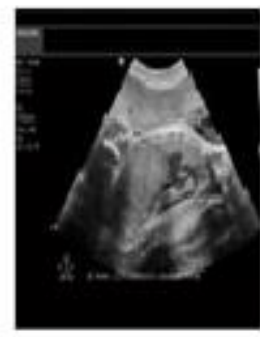

(a) Input image

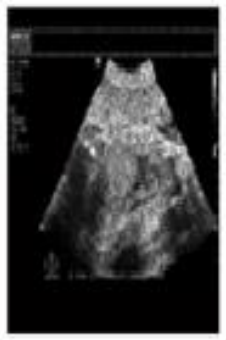

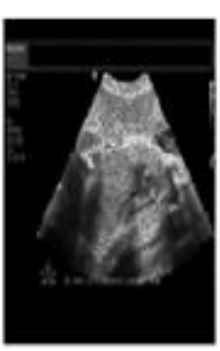

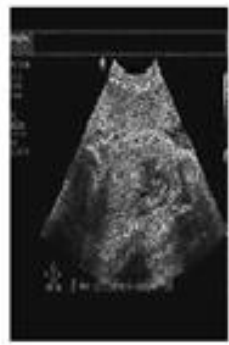

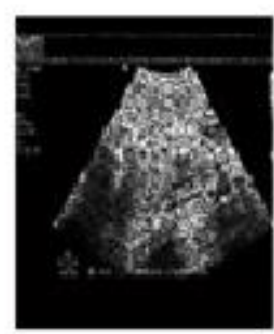

(b) Corrupted image

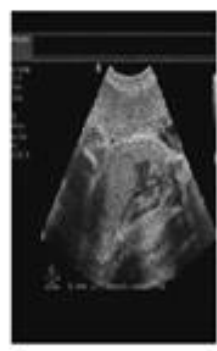

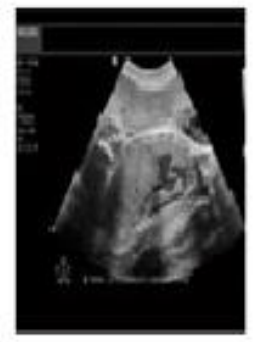



(c) AMLF
(d) AMKF
(e) AMBF
(f) AMEELF
(g) PSO EEMLF

Fig 3. Simulation results of different despeckling algorithm on Real medical image at noise density 40\% (a) Input Image (b) Corrupted image (c) AMLF (d) AMKF (e) AMBF (f) AMEELF (g) PSO EEMLF

The Quantitative analysis has been done in terms of Peak to Signal Noise Ratio (PSNR), Root Mean Square Error (RMSE), Structural Similarity Index Measure (SSIM), Mean Absolute Error (MAE) and Correlation Quality (CQ). The Table 1 to 5 represents the corresponding quantitative values.

Table 1. Comparison of PSNR values for different despeckling filter at noise density $40 \%$

\begin{tabular}{|l|c|c|c|c|c|}
\hline \multicolumn{1}{|c|}{ Images } & AMLF & AMKF & AMBF & AMEELF & PSO EEMLF \\
\hline Cyst 1 & 28.56 & 29.25 & 32.60 & 46.79 & 50.13 \\
\hline Cyst 2 & 30.12 & 32.60 & 35.20 & 51.85 & 52.40 \\
\hline Cyst 3 & 28.20 & 30.50 & 33.50 & 46.86 & 45.96 \\
\hline bt 1 & 27.96 & 29.65 & 32.60 & 47.2 & 46.25 \\
\hline bt 2 & 27.60 & 29.36 & 32.86 & 47.8 & 50.90 \\
\hline bt 3 & 28.23 & 30.12 & 33.12 & 49.06 & 51.62 \\
\hline SAR 1 & 27.12 & 28.95 & 30.18 & 46.18 & 47.36 \\
\hline SAR 2 & 27.50 & 29.50 & 31.01 & 46.49 & 47.51 \\
\hline
\end{tabular}

Table 2. Comparison of RMSE values for different despeckling filter at noise density $40 \%$

\begin{tabular}{|l|c|c|c|c|c|}
\hline \multicolumn{1}{|c|}{ Images } & AMLF & AMKF & AMBF & AMEELF & PSO EEMLF \\
\hline Cyst 1 & 4.44 & 3.64 & 2.59 & 1.159 & 0.766 \\
\hline Cyst 2 & 3.68 & 2.79 & 1.5 & 0.63 & 0.590 \\
\hline Cyst 3 & 3.41 & 3.2 & 2.51 & 1.12 & 1.23 \\
\hline bt 1 & 4.12 & 3.6 & 2.5 & 1.07 & 1.19 \\
\hline bt 2 & 4.63 & 3.55 & 2.49 & 1.00 & 0.70 \\
\hline bt 3 & 4.89 & 3.2 & 1.82 & 0.867 & 0.645 \\
\hline SAR 1 & 5.01 & 3.54 & 2.89 & 1.20 & 1.05 \\
\hline SAR 2 & 5.23 & 3.69 & 2.53 & 1.16 & 1.03 \\
\hline
\end{tabular}

Table 3. Comparison of SSIM values for different despeckling filter at noise density $40 \%$

\begin{tabular}{|l|c|c|c|c|c|}
\hline \multicolumn{1}{|c|}{ Images } & AMLF & AMKF & AMBF & AMEELF & PSO EEMLF \\
\hline Cyst 1 & 0.699 & 0.701 & 0.777 & 0.881 & 0.941 \\
\hline Cyst 2 & 0.68 & 0.70 & 0.754 & 0.879 & 0.926 \\
\hline Cyst 3 & 0.689 & 0.691 & 0.712 & 0.877 & 0.910 \\
\hline bt 1 & 0.701 & 0.752 & 0.788 & 0.871 & 0.911 \\
\hline bt 2 & 0.688 & 0.714 & 0.756 & 0.892 & 0.931 \\
\hline bt 3 & 0.702 & 0.754 & 0.772 & 0.899 & 0.925 \\
\hline SAR 1 & 0.687 & 0.692 & 0.712 & 0.889 & 0.935 \\
\hline SAR 2 & 0.682 & 0.689 & 0.715 & 0.881 & 0.929 \\
\hline
\end{tabular}

Table 4. Comparison of MAE values for different despeckling filter at noise density $40 \%$

\begin{tabular}{|l|c|c|c|c|c|}
\hline \multicolumn{1}{|c|}{ Images } & AMLF & AMKF & AMBF & AMEELF & PSO EEMLF \\
\hline Cyst 1 & 0.07 & 0.065 & 0.06 & 0.051 & 0.055 \\
\hline Cyst 2 & 0.067 & 0.053 & 0.052 & 0.049 & 0.040 \\
\hline Cyst 3 & 0.071 & 0.065 & 0.061 & 0.056 & 0.06 \\
\hline bt 1 & 0.074 & 0.069 & 0.064 & 0.0578 & 0.062 \\
\hline bt 2 & 0.055 & 0.046 & 0.030 & 0.025 & 0.04 \\
\hline bt 3 & 0.056 & 0.051 & 0.046 & 0.041 & 0.031 \\
\hline SAR 1 & 0.4 & 0.31 & 0.1 & 0.099 & 0.11 \\
\hline SAR 2 & 0.45 & 0.31 & 0.11 & 0.097 & 0.09 \\
\hline
\end{tabular}

Table 5. Comparison of CQ values for different despeckling filter at noise density $40 \%$

\begin{tabular}{|l|c|c|c|c|c|}
\hline \multicolumn{1}{|c|}{ Images } & AMLF & AMKF & AMBF & AMEELF & PSO EEMLF \\
\hline Cyst 1 & 0.575 & 0.58 & 0.61 & 0.65 & 0.75 \\
\hline Cyst 2 & 0.683 & 0.71 & 0.73 & 0.75 & 0.72 \\
\hline Cyst 3 & 0.64 & 0.668 & 0.69 & 0.72 & 0.69 \\
\hline bt 1 & 0.691 & 0.711 & 0.721 & 0.755 & 0.69 \\
\hline bt 2 & 0.632 & 0.67 & 0.68 & 0.69 & 0.695 \\
\hline bt 3 & 0.631 & 0.654 & 0.665 & 0.68 & 0.71 \\
\hline
\end{tabular}




\begin{tabular}{|l|l|l|l|l|l|}
\hline SAR 1 & 0.562 & 0.571 & 0.613 & 0.63 & 0.73 \\
\hline SAR 2 & 0.559 & 0.574 & 0.602 & 0.632 & 0.73 \\
\hline
\end{tabular}

The Corresponding figure for comparison for PSNR, RMSE, SSIM, MAE and CQ is shown in Fig 4.

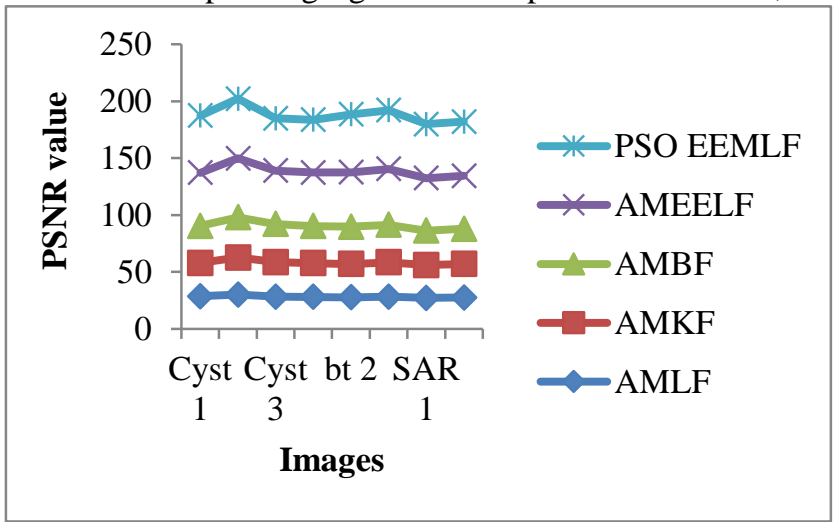

(a) PSNR Indication

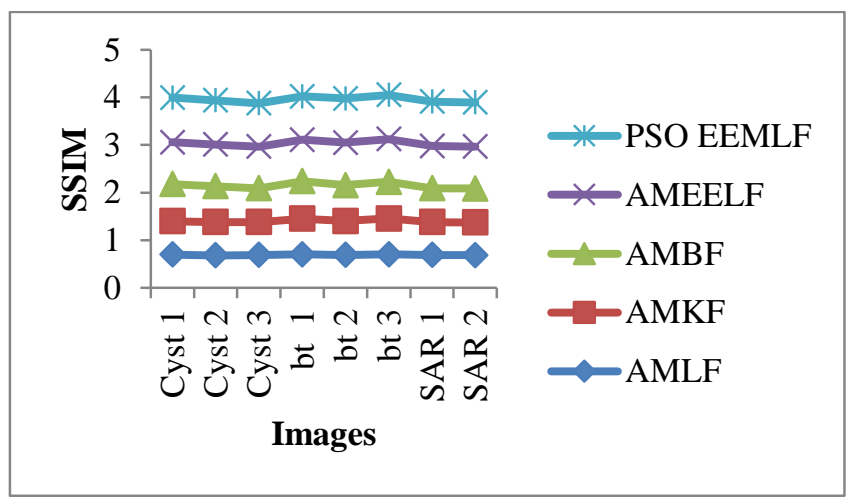

(c)SSIM Indication

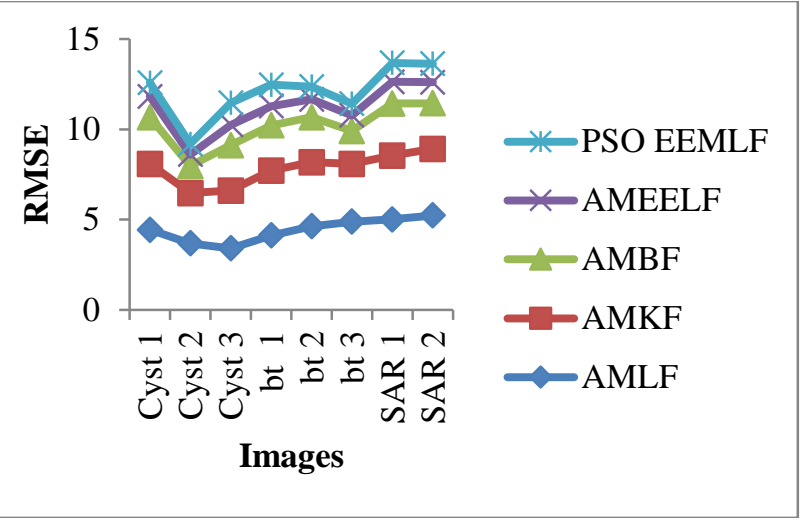

(b) RMSE Indication

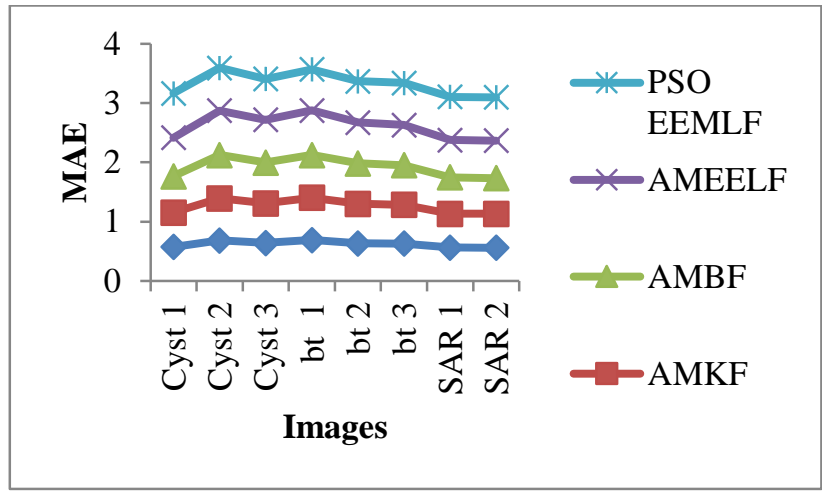

(d) MAE Indication

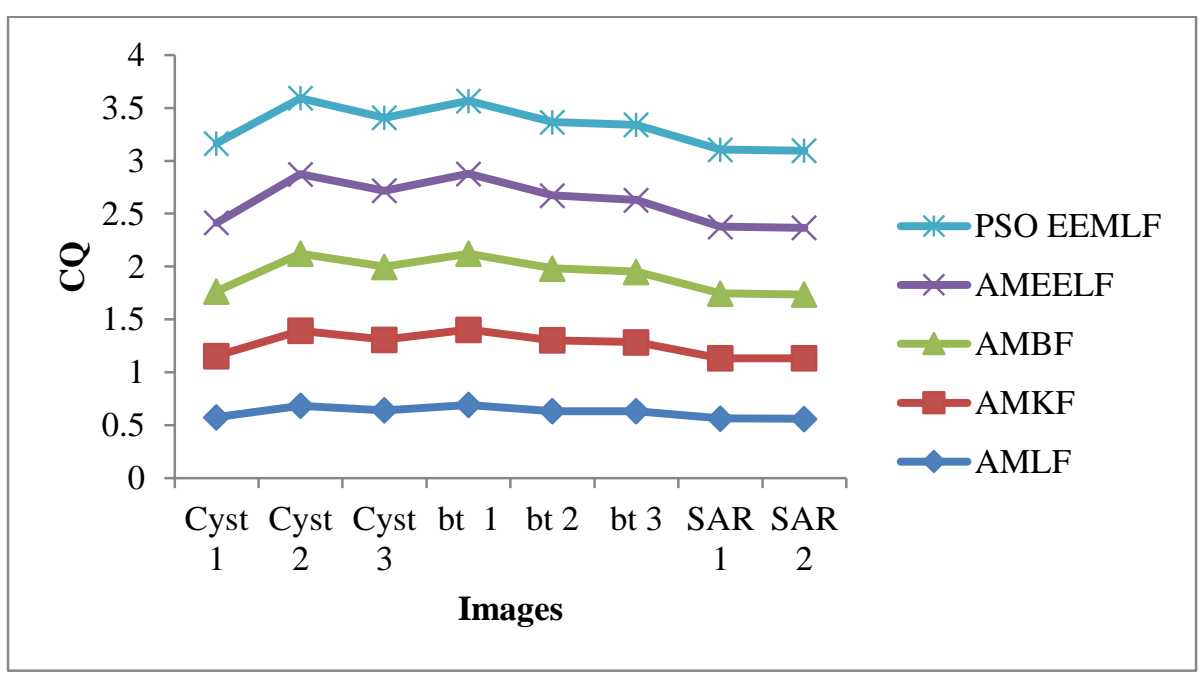

\section{(e) CQ Indication}

Fig 4. Graphical representation of Comparison of performance metrics

The comparison shows that the optimized algorithm with Edge Enhanced Modified Lee filter shows better results in terms of both quantitative and qualitative measures.

\section{Conclusion}

For removing speckle noise, the adaptive filters with modification are proposed. The optimization technique is also included along with the adaptive filters. The proposed despeckling filters like Adaptive Modified Lee filter, Adaptive Modified Kuan Filter, Adaptive Modified Bilateral filter, Adaptive Modified Edge Enhanced Filter and PSO based Edge enhanced Modified Lee filter are verified in both real and simulated 
images. The results are compared in terms of both Quantitative and Qualitative measures, in which the optimization based Edge Enhanced Lee filter gives good results in terms of PSNR and SSIM. Thus introducing optimization technique the performance of the filter is improved. The algorithms have been analyzed for different images for varying noise density from 20 to $80 \%$. Thus in the future optimization may be introduced for all adaptive filters which show better results.

\section{ACKNOWLEDGEMENT}

We thank the Management and Principal of Sri Ramakrishna Engineering College, Coimbatore, for providing the facilities and research support in carrying out the project in our Department.

\section{REFERENCES}

Darwin T Kuan, Alexander A Sawchuk, Timothy C Strand\&Pierre Chavel 1985, 'Adaptive noise smoothing filter for images with signal dependent noise. IEEE Transactions on Pattern Analysis and Machine Intelligence, vol.7, no.2, pp. 165-177.

Devasena, D\& Jagadeeswari, M 2017, 'FPGA Implementation of Speckle noise removal in Real Time Medical Images', Journal of Medical imaging and Health Informatics, vol. 7, no. 6, pp. 1263-1270.

Devasena D, Jagadeeswari M, Srinivasan K \& Radhika V, 'Hybrid Filter For Removal of Noise in Digital Images', International Journal of Recent Technology and Engineering, vol. 7, no. 6, pp. 1561-1564..

Dicom Example Files, MATLAB Central, Mathworks.

Hiremath, PS, Prema T Akkasaligar \& Sharan Badiger 2011, 'Speckle Reducing Contourlet Transform for Medical Ultrasound Images', International Journal of Computer and Information Engineering, vol.5, no.8, pp. 932-939.

Jong-Sen Lee 1980, 'Digital Image Enhancement and Noise Filtering by Use of Local Statistics', IEEE Transactions on Pattern Analysis and Machine Intelligence, vol.2, no. 2, pp.165-168.

Ju, C \& Moloney, C 1998, 'An Edge Enhanced Modified Lee filter for the Smoothing of SAR image speckle noise', Proceedings of IEEE workshop Nonlinear Signal Image Processing.

Loupas, T, Mcdicken, WN \& Allan, PL 1989, 'An Adaptive Weighted Median Filter for Speckle Suppression in Medical Ultrasonic Images', IEEE Transactions on Circuits and Systems, vol. 36, no. 1, pp.129-135.

Stian Solbo \& Torbjorn Eltoft 2008, 'A Stationary Wavelet-Domain Wiener Filter for Correlated Speckle', IEEE Transactions on Geoscience and Remote Sensing, vol. 46, no. 4, pp. 1219-1230.

Shivakumara Swamy, PM \& Vani, K 2015, 'A novel thresholding technique in the curvelet domain for improved speckle removal in SAR images', Optix, vol.127, no.2, pp.634-637.

Somnath Mukhopadhyay \& Mandal, JK 2014, 'Algorithms for Denoising Medical and Digital Images', Elesiver, pp.1-11. 